\title{
SCIENCE DE L'HOMME ET «DOMINATION RATIONNELLE » SAVOIR ETHNOLOGIQUE ET POLITIQUE INDIGÈNE EN AFRIQUE COLONIALE FRANÇAISE ${ }^{1}$
}

\author{
Benoît DE L'Estolle
}

\begin{abstract}
RÉsumÉ : L'article explore la notion wébérienne de «domination rationnelle » comme outil pour analyser les rapports entre les efforts de gestion des populations indigènes par l'État colonial, et le développement d'une forme spécifique de rationalité scientifique, prenant pour objet ces populations : les savoirs anthropologiques. En s'appuyant sur le cas de l'Afrique coloniale française, on s'efforce de montrer comment les dimensions d'instrumentation (avec la production d'instruments d'identification et de compréhension) et de légitimation sont essentielles dans l'émergence de savoirs sur les sociétés et les cultures indigènes, d'abord au sein des appareils administratifs, mais aussi dans les institutions savantes métropolitaines. L'appui au développement de la science de l'homme se fait dans le cadre de projets de «rationalisation» de la colonisation qui rapprochent des savants et des réformateurs liés à l'École coloniale, voulant redéfinir la profession d'administrateur colonial comme « spécialiste des indigènes ».
\end{abstract}

Mots-CLÉs : domination rationnelle, bureaucratie, Afrique coloniale française, histoire de l'anthropologie, politique indigène.

Summary : This paper explores the uses of the Weberian notion of « rational domination » as a tool for analysing the relationships between efforts by the Colonial State at controlling native populations and the development of a specific form of scientific rationality, taking these populations as objects : anthropological knowledge. Focusing on French colonial Africa, it argues that the aspects of instrumentation (with the production of instruments of identification and understanding) and of legitimization play an essential part in the emergence of a knowledge on native societies and cultures, firstly within the administration, but also in metropolitan scientific institutions. Support for the development of the science of man takes place within the framework of projects or "rationalization » of colonial domination that draw together scientists and reformers attached to the Colonial School, aiming at professionalizing the role of «colonial administrator », redefining it as a «specialist of natives».

KEYWORDS : rational domination, bureaucracy, French colonial Africa, history of anthropology, native policy.

1. Je remercie Jean-Claude Combessie, Adam Kuper, Federico Neiburg et Lygia Sigaud pour leurs commentaires sur une version antérieure de ce texte, présentée en 1997 au colloque de Rio.

Revue de synthèse : $4^{\mathrm{e}}$ S. $\mathrm{n}^{\text {os }}$ 3-4, juil.-déc. 2000, p. 291-323. 
ZusAmmenFASSUNG : In dem Artikel geht es um den Weberschen Begriff der rationalen Beherrschung als Werkzeug zur Analyse der Beziehungen zwischen den Bemühungen des Kolonialstaates, die einheimischen Bevölkerungen zu kontrollieren, und der Entwicklung einer spezifischen Form wissenschaftlicher Rationalität, deren Objekt diese Bevölkerungen war: der anthropologischen Erkenntnis. Am Beispiel der französischen Kolonien in Afrika soll gezeigt werden, welche entscheidende Rolle die Instrumentalisierung (verbunden mit der Erzeugung von Identifikations- und Verständnisinstrumenten) und die Legitimierung bei der Entstehung von Wissen über die einheimischen Gesellschaften und Kulturen gespielt haben. Das geschah zunächst innerhalb des Verwaltungsapparats, später auch in den gelehrten Institutionen. Im Rahmen der Pläne zur «Rationalisierung » der Kolonisation wurde auch die Wissenschaft des Menschen gefördert, wobei Wissenschaftler und Reformanhänger aus dem Umfeld der École coloniale gemeinsam versuchten, den Beruf des Verwaltungsexperten für die Kolonien als «Spezialist für Eingeborene » neu zu definieren.

STICHWÖRTER: nationale Beherrschung, Bürokratie, französische Kolonien in Afrika, Geschichte der Anthropologie, Eingeborenenpolitik.

RESUMo : O artigo explora a noção weberiana de «dominação racional » como instrumento para analisar as relações entre as práticas de gestão de populações nativas pelo Estado colonial e o desenvolvimento de uma forma especifica de racionalidade científica, tomando por objeto essas populações : os saberes antropológicos. A partir do caso da África colonial francesa, procura-se mostrar como as dimensões de instrumentação (com a produção de técnicas de identificação e de compreensão) e de legitimação são essenciais na emergência de saberes sobre as sociedades e as culturas nativas, tanto no interior dos aparelhos administrativos, como também nas instituições científicas metropolitanas. O apoio ao desenvolvimento da Ciência do Homem se realiza no âmbito de projetos de racionalização da colonização que aproximam ao mesmo tempo cientistas e reformadores ligados à Escola Colonial, interessados em redefinir a profissão de administrador colonial como «especialista em populações indígenas».

PALAVRAS-CHAVES : dominação racional, burocracia, África colonial francesa, história da antropologia, política indígena.

Benoît DE L'EsTolLE, né en 1967, enseigne l'anthropologie sociale et la sociologie à l'École normale supérieure. Il achève une thèse de doctorat à l'École des hautes études en sciences sociales qui compare le développement des savoirs anthropologiques sur l'Afrique coloniale en France et en Grande-Bretagne dans l'entre-deux-guerres. Dans le cadre d'un programme de coopération avec les anthropologues du Musée national de Rio de Janeiro, il coorganise aussi une enquête de terrain sur les transformations sociales en cours au Nordeste du Brésil avec le processus de Réforme agraire.

Adresse: Département de sciences sociales, École normale supérieure, 48 bd Jourdan, F-75014 Paris.

Courrier électronique : estoileb@elias.ens.fr 
«L'administration bureaucratique signifie essentiellement domination par le savoir. C'est le trait qui la rend spécifiquement rationnelle. »

Max WeBer

«La science au service de la colonisation aboutit toujours à l'homme, à la population, au milieu indigène. La grande science coloniale, c'est encore en définitive la science de l'homme. »

Gouverneur général Jules BRÉVIÉ

Les travaux sur les rapports entre les sciences sociales et l'État tiennent généralement pour acquis l'existence d'une contradiction fondamentale entre ces deux univers, qui peut être temporairement masquée par des alliances contre nature, mais finit toujours par resurgir, le progrès scientifique étant par essence lié à celui de l'autonomie à l'égard de tout pouvoir. Dans le cas des savoirs anthropologiques en particulier, leur relation avec des pratiques de gestion des populations semble ne pouvoir être abordée que sur le mode négatif de la dénonciation. Une autre piste sera suivie ici, inspirée par Bronislaw Malinowski, quand il formulait un programme de «rationalisation de l'anthropologie et de l'administration ${ }^{2}$ », indiquant ainsi la possibilité d'une affinité, au moins partielle, entre rationalité administrative et rationalité scientifique. Pour explorer cette hypothèse, une analyse sera engagée sur deux plans : théoriquement, en essayant de mettre en évidence des instruments d'analyse à partir de l'idée wébérienne que la forme spécifiquement moderne de la domination est caractérisée par le savoir et par la rationalité; empiriquement, en tentant d'utiliser ces instruments pour analyser les relations entre le développement des savoirs sur les populations indigènes et l'État colonial, à partir du cas de l'Afrique sous domination française entre 1920 et les années cinquante ${ }^{3}$.

Pour percevoir cette affinité, il faut d'abord s'extraire des schèmes liés à la spécialisation et à la division du travail entre disciplines, et mettre en relation des textes souvent classés aujourd'hui dans des catégories différentes, les uns relevant de «l'histoire de l'ethnologie ${ }^{4}$ », les autres de

2. Pour plus de précisions sur les références citées en notes, se reporter à la liste finale, p. 320-323. Ici, Malinowski, 1930. Ce texte (trad. en français in L'Estoile, 1994) propose tout à la fois un programme de réforme de l'administration coloniale et de révolution scientifique en anthropologie autour d'un commun «intérêt à la rationalisation ».

3. L'ethnologie américaniste française se développe dans un contexte de «colonisation intérieure » menée par d'autres États nationaux. Pour une analyse du contexte brésilien, où s'insèrent Claude Lévi-Strauss et Roger Bastide, voir Souza LimA, 1995, et l'article de Luiz Fernando Dias Duarte dans ce numéro, infra p. 325-344.

4. Le terme «ethnologie » correspond à la dénomination s'imposant progressivement en France au cours de cette période pour désigner l'ensemble de la science de l'homme qui 
« l'histoire coloniale ». On peut prendre, par exemple, le texte suivant, qui date de 1925 :

«Quand une colonie comprend des populations de civilisation inférieure ou très différente de la nôtre, de bons ethnologistes peuvent lui être aussi nécessaires que de bons ingénieurs, de bons forestiers ou de bons médecins. [...] Pour la mise en valeur, aussi complète et aussi économique que possible, de nos colonies, tout le monde reconnaît qu'il ne faut pas seulement des capitaux. Il faut aussi des savants, des techniciens, qui dressent un inventaire méthodique de leurs richesses naturelles (mines, forêts, cultures, etc.) et indiquent les procédés d'exploitation les meilleurs. La première de ces richesses naturelles, celle sans laquelle on ne peut à peu près rien faire des autres, surtout dans les régions équatoriales ou tropicales, n'est-ce pas la population indigène? N'y at-il donc pas un intérêt capital à l'étudier, elle aussi, méthodiquement, à avoir une connaissance exacte et approfondie de ses langues, de ses religions, de ses cadres sociaux qu'il est si imprudent de briser à la légère? »

Ce texte range les « ethnologistes » ( sic) parmi les savants et les techniciens contribuant par la mise en ordre du monde colonial à l'entreprise d'exploitation rationnelle de la nature tropicale. La population indigène, classée parmi les «richesses naturelles », et présentée comme conditionnant l'exploitation des autres richesses, appelle ses propres spécialistes. À côté des ingénieurs et des forestiers, chargés de la maîtrise rationnelle de la nature, médecins et «ethnologistes » doivent prendre soin de la population indigène, les uns en soignant les corps, les autres en se préoccupant de comprendre les «civilisations». Une telle déclaration pourrait être lue aujourd'hui comme une violation flagrante des valeurs d'autonomie de la science. Ce qui rend une telle lecture problématique, c'est la position de son auteur, une des plus établies du monde universitaire français d'alors. Il s'agit de Lucien Lévy-Bruhl, professeur de philosophie à la Sorbonne, directeur de la prestigieuse Revue philosophique, proche du groupe durkheimien, et savant internationalement reconnu pour ses travaux sur la « mentalité primitive ».

Ce passage est extrait d'un texte qui présente l'Institut d'ethnologie de l'université de Paris récemment créé ${ }^{5}$. Les arguments de Lévy-Bruhl, secrétaire général de l'Institut, ont paru suffisamment convaincants aux gouvernements coloniaux pour qu'ils y consacrent des sommes non négli-

prend pour objet privilégié les populations «primitives » et « indigènes » : linguistique, ethnographie, anthropologie physique et préhistoire. Pour simplifier, je n'entrerai pas ici dans le détail des différentes dénominations et des définitions concurrentes du savoir qu'elles recouvrent. J'utilise le terme de «savoir anthropologique » pour désigner ce domaine de savoir diversement qualifié à l'époque d'ethnologie, anthropologie, ethnographie, folklore, etc., et dont les frontières, notamment avec la géographie, sont parfois incertaines.

5. Lévy-BruHL, 1925. 
geables. En 1929, par exemple, l'Institut reçoit 166000 francs de subventions des colonies, pour 10160 francs de ressources propres, soit un rapport de 16 à $1^{6}$. L'existence même de l'Institut d'ethnologie est donc absolument dépendante des subventions coloniales.

On peut rapprocher ce texte d'un autre plaidoyer pour une colonisation scientifique, postérieur de quelques années :

«Aujourd'hui, [...] la colonisation a commencé sa période scientifique [...] Ici aussi, il faut savoir pour agir et pour prévoir [...] La mise en valeur agricole de l'Afrique noire suppose des études agronomiques détaillées, des expériences poursuivies avec continuité et impartialité, mais aussi, et au même degré, la connaissance du milieu indigène, du paysan noir, de sa mentalité, de ses méthodes, de ses aptitudes ${ }^{7}$. »

Cette affirmation du rôle d'une science impartiale pour la mise en valeur coloniale n'est pas purement rhétorique : son auteur, Jules Brévié (18801964), gouverneur général de l'Afrique-occidentale française (AOF) de 1931 à 1937, jouera un rôle important dans la création d'institutions de recherche dans le monde colonial; il crée en 1937 l'Institut français d'Afrique noire (IFAN), puis en 1942 l'Office des recherches scientifiques coloniales, qui deviendra l'ORSTOM ${ }^{8}$.

Comment expliquer l'étrange parenté entre deux textes, dont les auteurs occupent des positions a priori fort éloignées, de l'élite universitaire à la haute administration coloniale? Il existe une réponse toute prête, qui a souvent été mobilisée dans les débats sur le thème « anthropologie et colonialisme » : les ethnologues se sont mis au service du colonialisme, auquel ils ont fourni des instruments de manipulation des colonisés. Cette similitude des discours serait ainsi l'indice d'une complicité objective. Selon une lecture opposée, la proximité serait purement tactique : des déclarations comme celles de Lévy-Bruhl (ou de Malinowski) ne seraient que de pures « courbettes rhétoriques » aux autorités coloniales, nécessaires pour que les chercheurs puissent poursuivre leurs «véritables» objectifs, «purement scientifiques $\gg{ }^{9}$.

Si l'on refuse les simplismes symétriques de la dénonciation ou de la dénégation, il nous faut comprendre ce que signifie ce rapprochement de savants reconnus et de coloniaux autour de la revendication d'une colonisation rationnelle, appuyée sur l'étude scientifique des populations indigènes.

6. LÉVY-BruHL, 1929, p. 421.

7. «Science et colonisation », extr. de la revue Science et industrie, repr. in BRÉvIÉ, 1936.

8. Office des recherches scientifiques et techniques outre-mer, récemment rebaptisé Institut de recherches pour le développement. Voir Bonneuil, 1991.

9. Voir, p. ex., Goody, 1995, p. 42-43. Voir l'analyse développée en introduction à ce numéro, supra p. 233-263. 
Pour explorer cette convergence, au moins partielle, entre rationalité administrative et rationalité scientifique, la notion de «domination rationnelle » fournit une piste.

\section{DOMINATION BUREAUCRATIQUE ET RATIONALISATION}

Une analyse des affinités entre rationalité administrative et rationalité scientifique peut en effet trouver chez Max Weber un certain nombre d'outils, en particulier dans son analyse de la «domination rationnelle », qu'exemplifie la bureaucratie ${ }^{10}$. Ce qui fait la richesse, mais aussi l'ambiguïté de l'analyse de la domination que propose Weber, c'est son caractère double : d'une part, sa typologie est explicitement construite non à partir des caractéristiques effectives de la domination, mais à partir du principe (fondement) de légitimité revendiqué pour cette domination; d'autre part, Weber propose en fait dans son analyse une caractérisation des traits formels des divers types de domination, traits qui n'apparaissent pas nécessairement déterminés par le principe de légitimité dominant. La notion de «domination rationnelle » peut donc être analysée en prenant en compte ces deux aspects.

Weber voit dans la domination bureaucratique la forme spécifiquement moderne de la domination, marquée en particulier par son caractère rationnel, calculable. Elle constitue l'accomplissement, du point de vue de la domination, du processus de rationalisation qui pour Weber est spécifique de l'Occident ${ }^{11}$. Dans cette acception, la notion de «domination rationnelle » prend essentiellement un sens formel, dans la mesure où la bureaucratie favorise une « rationalisation » des méthodes de domination. Ce qui fait de la domination bureaucratique une forme de domination « spécifiquement rationnelle », c'est précisément qu'elle est une domination fondée sur le savoir ${ }^{12}$.

Le besoin de savoir est donc inscrit dans les exigences de l'administration bureaucratique, et la production d'un savoir spécifique apparaît comme une des caractéristiques de cette forme de domination. Le déve-

10. Notamment in WEBER, 1971, «Les types de domination légitime », p. 217-226, et Weber, 1968, vol. II, chap. XI, « Bureaucracy », p. 956-1005. Voir aussi Weber, 1996, p. 369375. Notre lecture de Max Weber n'obéit pas à des préoccupations exégétiques, mais vise à dégager des instruments d'analyse.

11. WeBER, 1971, p. 223 : le type purement bureaucratique est, « d'un point de vue purement technique, capable d'atteindre le plus haut degré d'efficacité et est, en ce sens, formellement le plus rationnel des moyens connus d'exercer la domination sur des êtres humains ».

12. WEBER, 1971, p. 225. 
loppement de la domination bureaucratique, dans la mesure où elle met l'accent sur la compétence technique des fonctionnaires, promeut une formation spécialisée. La domination bureaucratique apparaît ainsi chez Weber étroitement associée à deux autres phénomènes : d'un côté, un processus de rationalisation; de l'autre, l'importance du savoir et de l'expertise, tant dans les pratiques administratives que dans la formation des agents ${ }^{13}$. La rationalisation des instruments de connaissance de son domaine d'intervention, favorisée par l'activité bureaucratique, se trouve ainsi en affinité avec la rationalisation des systèmes de production de connaissance qu'effectue l'activité scientifique ${ }^{14}$.

À ce premier niveau de lecture, le caractère rationnel est perçu comme un trait positif, qui caractérise cette forme de domination par opposition aux formes irrationnelles de domination charismatique ou traditionnelle. On peut cependant analyser aussi la domination de type «rationnel-légal» en accordant un rôle central aux notions associées de légitimation et de croyance. Dans l'analyse wébérienne de la domination, la croyance joue en effet un rôle central. La légitimité «traditionnelle » repose sur la croyance au caractère sacré de la tradition, de même que la légitimité charismatique est fondée sur la croyance au «don surnaturel» du leader charismatique. De la même façon, selon Weber, la légitimité de la domination légale repose sur la croyance dans son caractère «conforme à la légalité » et rationnel. Si l'on peut définir la forme spécifiquement moderne de la domination comme une «domination rationnelle », c'est au sens où sa légitimité repose sur la croyance au fait qu'elle est fondée en raison ${ }^{15}$. L'enjeu n'est donc pas, dans cette perspective, d'évaluer le caractère effectivement plus (ou moins) rationnel de la domination bureaucratique moderne (de même que le caractère réellement traditionnel de la «domination traditionnelle » n'est pas ce qui intéresse Weber), mais d'analyser ce que veut dire le fait qu'elle s'impose au nom de la raison. Weber invite ainsi à prendre au sérieux le discours de justification de la domination (plutôt que de le disqualifier a priori comme «idéologique»), parce qu'il a des effets sur les «croyances » de ceux qui sont impliqués dans la relation de domination, et sur la pratique de celle-ci.

De ce point de vue, on peut dire, en poursuivant l'analyse de Weber, que ce qui caractérise l'État moderne, c'est bien une revendication de rationalité. La « conformité à la raison » devient un principe de légitimité, c'est-à-

13. Weber, 1968, vol. II, p. 998 : «La bureaucratisation de toute domination favorise très fortement le développement de l'objectivité rationnelle et le type de personnalité de l'expert professionnel. »

14. WEBER, 1996, p. 492.

15. Weber, 1968, vol. II, p. 919 : «Le seul point décisif pour nous est qu'en principe un système de raisons rationnellement discutables se trouve derrière tout acte d'administration bureaucratique, que ce soit en le ramenant à des normes ou en pesant les moyens et les fins. » 
dire à la fois un argument de justification et un des critères essentiels d'évaluation du caractère légitime de la domination : c'est ce que l'on peut appeler le «paradigme des Lumières », reposant sur une alliance entre des spécialistes de la Raison et les modernisateurs de l'État monarchique ${ }^{16}$. La Science, qui apparait comme l'instance de rationalisation et de progrès par excellence, devient un élément de légitimation essentiel pour le pouvoir d'État, se substituant progressivement à la Religion. L'appui donné à la Science manifeste le fait que l'État se trouve bien du côté de la rationalité et que, de même qu'il a «le Droit pour lui », il a aussi «la Raison pour lui ».

Il est cependant fondamental de souligner que cette formulation commode en termes d' «État » et de « Science » ne renvoie pas à une opposition atemporelle entre deux univers clos en relation d'extériorité. Weber insiste, en particulier, sur la nécessité de prendre en compte le rôle des agents de l'État, qui ont un intérêt spécifique à la rationalisation formelle, dans la mesure où elle les protège contre l'arbitraire du pouvoir politique. À la vision de blocs monolithiques antagonistes, il apparaît plus fécond de substituer une conception de l'État comme un espace de concurrence entre groupes interdépendants, dans lequel des «spécialistes du savoir» (qui peuvent être ou non des agents de l'État) interviennent à la fois comme protagonistes et comme alliés potentiels ${ }^{17}$. De même, la notion de «Science» ne renvoie pas à une définition immuable de la science, mais à l'ensemble des pratiques reconnues commes «scientifiques » à un moment donné, dont les frontières sont un enjeu essentiel.

La notion de «domination rationnelle», telle que l'emploie Weber, permet donc une double lecture: d'une part, elle caractérise des formes de rationalisation des méthodes de domination; de l'autre, elle renvoie au principe de légitimation invoqué, c'est-à-dire à la revendication d'une domination qui s'affirme comme rationnelle.

Si l'on analyse la façon dont la science peut être mobilisée par la «domination rationnelle », on peut opposer ces deux dimensions : d'un côté, la science fournit à l'État et à ses agents des ressources pour une rationalisation des instruments d'orientation et de contrôle (instrumentation); de l'autre, elle peut être mobilisée dans des stratégies de légitimation ${ }^{18}$. Nombre d'analyses des rapports entre la rationalité scientifique, notamment sous la forme qu'elle prend dans les sciences sociales, et les formes modernes de domination, privilégient, au détriment de l'autre, l'une de ces deux dimensions, en insistant soit sur la dimension de légitimation,

16. BRIAN, 1994.

17. Cette vision s'appuie sur l'analyse faite par WEBER, 1996, p. 241-328, du rapport entre domination hiérocratique et domination politique.

18. Pour une première formulation, voir L'Estolle, 1997c, p. 348. 
soit sur celle d'instrumentation, en lien avec l'émergence de «technologies politiques ». Il semble plus fécond de poursuivre l'analyse en conservant le double sens que prend la notion de «domination rationnelle » chez Weber. Un des intérêts du recours à la notion de légitimation est qu'elle permet d'échapper à une analyse par trop exclusive en termes d'instrumentalisation ${ }^{19}$. Elle invite à dépasser l'opposition trop simple, et surchargée de jugements de valeur, entre un savoir utilitaire, directement produit à des fins instrumentales, et un savoir «inutile», présumé désintéressé, donc plus noble; dans une perspective de légitimation de la domination, un savoir «gratuit», non intéressé, peut se révéler plus «efficace » qu'un savoir trop visiblement dirigé vers des objectifs pratiques.

Ces deux dimensions sont idéal typiques: elles ne doivent pas être conçues comme des possibilités mutuellement exclusives, mais plutôt comme deux pôles entre lesquels prennent place les diverses pratiques savantes coloniales. Il est ainsi possible d'analyser la revendication de rationalisation de la connaissance des populations dans le contexte colonial non seulement du point de vue du développement d'un arsenal d'instruments de déchiffrement du monde colonisé, mais aussi du point de vue des discours de légitimation de la domination.

\section{IDENTIFICATION DES RACES ET COMPRÉHENSION DES INDIGÈNES}

C'est essentiellement dans sa dimension instrumentale qu'a été analysée l'entreprise de connaissance scientifique des territoires et populations soumis à la colonisation. Ainsi, l'effort de connaissance considérable, réalisé dès avant la conquête et pendant celle-ci par les explorateurs, puis par les militaires, a pu être interprété sur le mode du travail de renseignement qui fait partie de l'arsenal des technologies de conquête, puis de contrôle, des populations soumises et pacifiées ${ }^{20}$. Plus généralement, l'administration des territoires conquis implique un travail de rationalisation, au sens d'une mise en ordre du monde colonisé. À cette tâche d'orientation et de mise en ordre, les discours savants de l'anthropologie, dans leurs différentes versions, fournissent des instruments : ils proposent des critères d'identification des populations et des cadres d'interprétation qui permettent de donner une intelligibilité à des comportements perçus comme déroutants. Cette visée est souvent revendiquée; de fait, c'est d'abord en termes d'utilité pra-

19. Plutôt que le terme d'instrumentalisation, souvent utilisé dans une intention dénonciatrice, nous utiliserons ici le terme plus analytique d'instrumentation.

20. Nordman et Raison, 1980. 
tique que l'administration justifie la nécessité de produire des connaissances sur les régions et les populations qu'elle entend contrôler. La hiérarchie administrative recommande régulièrement la production de travaux de synthèse capitalisant, sous la forme de monographies, l'état du savoir sur les différentes circonscriptions ${ }^{21}$. Ainsi, en 1920, le commissaire de la République au Cameroun insiste sur l'urgence qu'il y a à connaître ce territoire, retiré à l'Allemagne et attribué à la France, sous mandat de la Société des nations, à l'issue de la Première Guerre mondiale. Il demande donc la rédaction de monographies, comprenant un chapitre sur l'ethnographie, pour lequel il propose le plan suivant :

«Ethnographie. Histoire - langages - coutumes — droit coutumier - religion - groupement de populations et commandement indigène - caractéristiques de la race - population — densité — habitat — formes de la vie familiale - vêtements ${ }^{22}$.»

L'identification de groupes de population différenciés, appelés généralement « races », et la nécessité d'adapter la politique à mener en fonction de leur spécificité est un thème récurrent dans les discours coloniaux, comme dans la correspondance administrative de l'entre-deux-guerres. La grille ethnique fournit un langage permettant le déchiffrement des populations. L'administration consacre des efforts importants à distinguer et à localiser les « groupes ethniques » (ce terme savant se substitue lentement à celui de « races »), catégorie de perception des différences sociales qui structure à la fois les pratiques administratives coloniales et les discours savants ${ }^{23}$. De fait, la production d'instruments d'identification et de classification des populations est un des domaines où se manifeste le mieux la convergence entre des formes d'objectivation administrative et d'objectivation scientifique, culminant dans la production d'une ethnologie au sens strict (s'attachant à reconstituer les origines et les migrations des ethnies). La production d'inventaires ethniques, donnant une évaluation chiffrée des différents groupes, et de cartes, visant à préciser leur répartition spatiale, constitue ainsi l'objet d'efforts répétés de l'administration coloniale ${ }^{24}$. Une des caractéristiques de ces travaux réside en leur capacité de circulation dans

21. La répétition même de ces demandes indique cependant qu'elles ne sont pas toujours suivies d'effet.

22. Archives de l'Agence économique du Cameroun (AEC), Aix-en-Provence, Centre des archives d'outre-mer (CAOM), Commissaire de la République au Cameroun, circulaire $\mathrm{n}^{\circ} 43$, 29 déc. 1920.

23. Voir, sur ce thème, les analyses réunies in Amselle et M'BокоLо, 1999, en part. Jean BAZIN, p. 87-127, « À chacun son Bambara », qui montre comment des pratiques classificatoires savantes et administratives, aboutissent à cristalliser et à réifier des catégories fluides de la société précoloniale.

24. Pour une comparaison avec le cas de l'Inde, voir CoHn, 1987. 
des espaces variés. Ils se veulent généralement orientés vers la pratique, tout en obéissant à des principes scientifiques : produits d'une rationalité administrative, ils peuvent aussi être publiés dans les revues savantes, ou encore utilisés à des fins de propagande.

Un rapport du Bureau des affaires politiques, daté du 30 octobre 1933, établit ainsi un «état des différents groupes linguistiques au Cameroun », qui en donne une estimation numérique et évalue les tendances hégémoniques de certains groupes de langue, notamment Fang et Douala ${ }^{25}$. Une telle étude prend sens dans la préoccupation de l'administration coloniale pour une réduction du nombre de langues africaines, qui apparaissent comme un obstacle à la communication, et pour la détermination des langues que doivent apprendre les administrateurs. Le même rapport est publié quelques mois plus tard dans le Journal de la Société des africanistes, organe de la société savante du même nom, sous le titre «Inventaire ethnique et linguistique du Cameroun sous mandat français ${ }^{26}{ }$. La même année, le Commissaire décide d'attribuer des primes aux administrateurs qui auront appris les langues indigènes, afin de les encourager à «pénétrer la mentalité de leurs administrés ${ }^{27}$ ».

Toujours au Cameroun, un des problèmes politiques identifiés par l'administration est constitué par la présence dans le Nord de populations « païennes » (dites Kirdis) sous la domination de groupes islamisés (Foulbés). L'administration décide en 1923-1924 de créer des cantons kirdis ethniquement homogènes, indépendants des Foulbés, et encourage l'étude de leurs « coutumes » par les administrateurs ${ }^{28}$. Dix ans plus tard, le directeur du Bureau des affaires politiques, Chauleur, rédige un long mémorandum consacré à La Région Nord, en préparation de la tournée que doit réaliser le commissaire de la République dans cette région. L'auteur précise que «si un accent spécial a été mis sur les tribus du Nord-Cameroun, c'est que la question politique est essentiellement dépendante des mouvements, relations et réactions des races diverses qui peuplent la région ».

Vingt-six pages sont consacrées aux races, pour lesquelles il donne une « répartition ethnique » chiffrée et des cartes ${ }^{29}$. Le même Chauleur rédige en 1935-1937, à partir des archives de son service, des travaux d'anthropologie physique du Dr Léon Poutrin et de travaux allemands, une «étude générale relative au peuplement du Cameroun », qui vise notamment à éta-

25. AEC, CAOM, 353/168 bis, «Population, recensement», rapport du Bureau des affaires politiques, 30 oct. 1933.

26. «Inventaire ethnique... », 1934

27. Arrêté du 21 avril 1933, Journal officiel des colonies, 1933, p. 244.

28. AEC, CAOM, 928/2903, «Monographies », dossier « Pacification Nord Cameroun », 1923. Voir aussi Rapport annuel du gouvernement français sur l'administration sous mandat des territoires du Cameroun pour 1924, 1925, p. 24.

29. AEC, CAOM, C888, « Monographies », La Région Nord, dactylogr., 145 p. 
blir les origines, les migrations et les mélanges des différents groupes; cette étude sera publiée en 1943 dans le Bulletin de la Société d'études camerounaises, sous le titre «Esquisse ethnologique pour servir à l'étude des principales tribus des territoires du Cameroun sous mandat français ${ }^{30}$ ».

Ces passages des bureaux de l'administration à des publications savantes illustrent la continuité entre des formes de savoir administratif et un discours scientifique. Il est significatif que ces préoccupations classificatoires soient reprises par les organismes de recherche qui se mettent progressivement en place. La transformation du Comité d'études historiques et scientifiques de l'Afrique occidentale française (AOF), société savante qui regroupait, sous la responsabilité du gouverneur général, les coloniaux intéressés en Institut français de l'Afrique noire, sous la direction de chercheurs détachés depuis la métropole, peut être lue comme une autonomisation progressive de l'appareil de production de connaissances par rapport à l'appareil administratif, et un rattachement aux centres scientifiques métropolitains. Pourtant, l'IFAN poursuit l'œuvre d'inventaire et d'identification des populations, en établissant dans ses différents centres des «fichiers ethniques » nourris par les données des administrateurs et des chercheurs. Au Cameroun, le travail de Chauleur, repris, à partir des archives du Bureau des affaires politiques, et enrichi par des rapports de tournée, des recensements et par les contributions des chefs de circonscription et des membres du service de santé, aboutit en 1949 à la publication d'un Inventaire ethnique du Sud-Cameroun ${ }^{31}$. La synthèse de ce savoir collectivement accumulé a été réalisée par Idelette Dugast, dont la trajectoire illustre bien la continuité entre savoir administratif et ethnologie universitaire ${ }^{32}$.

La même analyse vaut pour les cartes ethniques, instrument d'identification à la fois savant et administratif ${ }^{33}$. En 1939, le Journal de la Société des africanistes publie une «Carte schématique des populations du Cameroun », réalisée par un jeune ethnographe, membre de la quatrième mission Griaule, dite «Sahara-Cameroun», (et futur pionnier de la recherche urbaine), Paul-Henry Chombart de Lauwe, et par un administrateur-chef

30. Dugast, 1949, p. vi; Dugast, 1948.

31. Il inaugure une série des « Mémoires de l'Institut français d'Afrique noire » (Centre du Cameroun) consacrée aux «Populations », voir Dugast, 1949, introd.

32. Élève dans les années trente de l'Institut d'ethnologie, qui l'envoie en mission au Cameroun, elle y épouse l'administrateur-chef des Colonies René Dugast; en 1943, elle devient secrétaire-archiviste de la Société d'études camerounaises, qui se transforme l'année suivante en un centre local de l'IFAN. Elle contribue également à la vaste entreprise de synthèse systématique coordonnée par l'Institut africain international dans le cadre de l'Ethnographic Survey of Africa, voir Dugast, 1954; elle est, enfin, l'auteur d'une Monographie de la tribu des Ndiki (Banen du Cameroun), voir Dugast, 1955-1959.

33. La revue Togo-Cameroun, consacrée à la «propagande » pour les territoires sous mandat, publie régulièrement des «cartes ethnographiques » du territoire, également présentes dans les Rapports annuels du gouvernement français sur l'administration sous mandat des territoires du Cameroun. 
des colonies, J. Deboudaud ${ }^{34}$. Les auteurs précisent la visée identificatoire qui a présidé à l'élaboration de cet «instrument provisoire », et ses conséquences sur la méthodologie adoptée :

«L'intérêt actuel d'une carte de répartition n'est pas d'illustrer une classification plus ou moins arbitraire, mais de noter les principales distinctions utiles à l'Administration.

« Aussi tous les caractères sont bons pour définir et délimiter un groupe ethnique, principalement les coutumes matérielles, politiques et religieuses plus encore que les données géographiques et linguistiques dont nous parlions précédemment ${ }^{35}$.»

Ainsi, les cartes ethnographiques sont conçues autant comme des outils de gestion administrative que comme des instruments de totalisation savante. L'effort de production de ces cartes, d'abord réalisé au sein de l'administration, fait progressivement l'objet d'une délégation à des chercheurs professionnels, géographes et ethnologues, qui compléteront et systématiseront le travail des administrateurs, et sera pris en charge après 1945 par l'IFAN ${ }^{36}$.

Au-delà de la visée identificatoire, la pratique de l'ethnographie est conçue comme un effort de compréhension des populations «primitives» ou « attardées ». Ce qui est remarquable, c'est qu'un savoir d'origine largement administrative peut être validé dans un contexte savant, c'est-à-dire obtenir la reconnaissance de son caractère de document pour la science. De fait, une des caractéristiques essentielles de ce savoir colonial est l'absence d'étanchéité entre des domaines qui, pour des universitaires de la fin du $\mathrm{xx}^{\mathrm{e}}$ siècle, sont (en principe) nettement séparés. Il y a plutôt un va-et-vient constant entre savoir administratif et ethnographie. La monographie de l'administrateur Félix Éboué constitue un exemple particulièrement significatif de cette continuité entre savoir «colonial» et savoir «scientifique ${ }^{37}$, à la fois dans la mesure où il est présenté à l'époque comme «exemplaire » et en raison de la carrière exceptionnelle de son auteur ${ }^{38}$. Faisant d'abord l'objet d'une communication à la section d'Ethnographie du congrès d'Anthropologie et d'Archéologie préhistorique, pendant

34. Chombart de Lauwe et Deboudaud, 1939.

35. Chombart de Lauwe et Deboudaud, 1939, p. 197 (souligné par moi).

36. Voir, notamment, la série de cartes ethno-démographiques de l'Afrique occidentale, au 1/100 000, publiées par l'IFAN de Dakar.

37. ÉвоUÉ, 1931.

38. Félix Éboué (1884-1944), d'origine guyanaise, noir, diplômé de l'École coloniale en 1908, reste de longues années administrateur dans une colonie située au dernier rang de la hiérarchie coloniale, l'Oubangui-Chari (République centrafricaine actuelle); il deviendra par la suite gouverneur général du Tchad, puis de l'AEF, mettant en place une nouvelle « politique indigène » fondée sur le respect des institutions politiques et des coutumes traditionnelles. Il est aussi le seul «colonial» entré au Panthéon. 
l'Exposition coloniale de 1931, il est ensuite publié dans plusieurs revues, coloniales et savantes ${ }^{39}$. Il est, enfin, édité en brochure séparée par les soins du Gouvernement général.

Le gouverneur général Raphaël Antonetti, représentant de la haute hiérarchie coloniale, invite ses subordonnés à réaliser un tel travail de connaissance dans leur circonscription ${ }^{40}$. Une circulaire, au caractère très officiel, puisqu'elle est envoyée à tous les administrateurs et publiée au Journal officiel de l'Afrique équatoriale française, donne en modèle la monographie d'Éboué, dont un exemplaire est adressé à chaque poste.

«Le remarquable travail publié par M. Éboué pose d'ailleurs une question d'ordre plus général qui intéresse tous ceux qui sont appelés à commander dans la brousse. Je ne cesserai de rappeler qu'une bonne administration doit être fondée sur une connaissance aussi complète que possible de nos indigènes. « Rien ne saurait remplacer cette connaissance et c'est pourquoi j'aimerais voir les Administrateurs se consacrer, dans la mesure du possible, à des enquêtes analogues à celle de M. Éboué, en cherchant dans une compréhension plus approfondie de leurs indigènes moins un exercice de la curiosité ou un délassement intellectuel que le moyen d'éviter, grâce à elles, bien des erreurs, en même temps qu'ils trouveraient la possibilité d'user de ces coutumes mêmes pour faire entendre dans leur propre langue, en quelque sorte, à leurs administrés ce que nous attendons d'eux ${ }^{41}$. »

Le travail ethnographique constitue donc une forme d'excellence, présentée en modèle à tous les administrateurs ${ }^{42}$. Il s'agit de maîtriser la «langue » (on dirait aujourd'hui le «code») des sociétés administrées. La connaissance systématique et l'effort de compréhension des indigènes apparaissent ici comme des outils de communication et de commandement que doit maîtriser l'administrateur pour accomplir sa tâche.

Dans le même temps, la brochure est envoyée par Raphaël Antonetti aux représentants de l'ethnologie métropolitaine, notamment à Henri Labouret, qui enseigne l'ethnographie à l'École coloniale et à l'Institut d'ethnologie, et à Lucien Lévy-Bruhl. Les remerciements de ce dernier, qui dit sa reconnaissance au Gouverneur général pour son appui à l'Institut d'ethnologie, sont significatifs :

39. Il paraît ainsi à la fois dans le supplément du Bulletin de l'Afrique française. Renseignements coloniaux et documents, dans la revue d'une société savante locale, le Bulletin de la Société des recherches congolaises, publ. par le Gouvernement général de l'Afrique équatoriale française, et dans la revue d'une société savante métropolitaine, L'Ethnographie.

40. Raphaël Antonetti (1872-1938) est gouverneur général de l'AEF de 1924 à 1934.

41. Archives du Gouvernement général de l'Afrique équatoriale française (GGAEF), CAOM, 5D103, circulaire du gouverneur général Antonetti, 16 oct. 1933.

42. Sa reconnaissance en tant qu'ethnographe, accomplissant une des formes de l'excellence de l'administrateur (la compréhension des indigènes), a signalé Éboué à l'attention de ses supérieurs. 
« J'ai lu avec le plus grand intérêt ces observations sur les peuples de l'Oubangui-Chari par un homme qui sait voir, et qui semble avoir une longue expérience de ces populations. Jamais les lumières que l'ethnographie peut procurer sur la mentalité des indigènes n'ont été plus précieuses qu'aujourd'hui. Il est heureux que nos administrateurs sentent de plus en plus l'utilité, on pourrait dire la nécessité de ces études, et y apportent eux-mêmes tout le concours qu'ils peuvent ${ }^{43}$.»

De façon plus générale, l'encouragement de la haute hiérarchie administrative à se livrer à des études sur « leurs » populations semble devenir plus net dans les années trente, au moins dans certains territoires. Cet accent mis sur la compréhension correspond aussi à une inquiétude : après le soulèvement de Yen-Bay en Indochine, attribué au manque de contacts entre l'administration et les populations indigènes, une circulaire du ministère des Colonies recommande en 1930 aux administrateurs de tous les territoires de se tenir en contact plus étroit avec les populations qu'ils administrent, de multiplier leurs tournées et de porter un intérêt personnel plus grand aux indigènes ${ }^{44}$. On trouve ainsi dans la correspondance administrative des encouragements très fermes à réaliser des travaux ethnographiques $^{45}$.

La dimension de «technologie politique », dans une visée de maîtrise et de transformation des sociétés indigènes, apparaît donc comme fondamentale pour comprendre l'investissement de certains agents de l'État colonial dans le savoir ethnographique. Celui-ci fournit à la fois des outils d'identification et des cadres interprétatifs et contribue ainsi à la production d'instruments formellement rationnels de «mise en ordre » du monde colonisé, qui engendrent un sentiment rassurant de contrôle d'une réalité complexe. Il faut pourtant préciser que cette volonté de savoir et de «mise en ordre » doit tenir compte des stratégies des colonisés et peut n'aboutir qu'à une apparence d'ordre ${ }^{46}$. S'il y a donc bien une certaine continuité entre des formes de rationalité administrative et de rationalité scientifique, cette dimension «instrumentale » n'épuise pourtant pas les usages coloniaux du savoir anthropologique.

43. GGAEF, CAOM, 5D103, lettre de Lucien Lévy-BRuHL au gouverneur général de l'AEF, 24 oct 1933.

44. Circulaire du 10 juin 1930, Bulletin officiel des colonies, citée par CoHEN, 1973, p. 191.

45. GGAEF, CAOM, Bureau des affaires politiques, passim. D'autres témoignages font cependant état d'un manque de compréhension pour les travaux ethnographiques, voir CoHEN, 1973, p. 184-185.

46. Sur l'instabilité de ces constructions, voir Pels, 1996. 
CRISE DE LA DOMINATION ET « SUPÉRIORITÉ SCIENTIFIQUE »

L'accent nouveau mis sur l'effort nécessaire de connaissance et de compréhension des indigènes doit aussi être mis en relation avec une transformation plus générale des conditions de la domination coloniale et, en particulier, des nouvelles nécessités de sa légitimation sur la scène internationale. La présence dans les pratiques savantes d'une dimension de légitimation n'est certes pas nouvelle : auparavant, elle donnait déjà à l'ethnographie un sens dépassant la simple visée instrumentale. Depuis le $\mathrm{XIX}^{\mathrm{e}}$ siècle, le savoir anthropologique joue, en effet, un rôle essentiel dans la formulation et la justification de la «mission civilisatrice » dans la mesure où il apporte une caution scientifique au cadre d'interprétation évolutionniste $^{47}$. L'ethnographie apparaît ainsi, parmi les pratiques savantes coloniales, comme complémentaire de la chronique de la conquête coloniale : souvent associée à l'archéologie, elle présente une sorte de "point-zéro », de «pré-histoire », qui documente l'état des peuples indigènes avant leur « entrée dans l'histoire », commençant avec la conquête elle-même, premier acte d'un processus de civilisation impulsé de l'extérieur. Ce schéma évolutionniste structure la plupart des présentations ethnographiques dans les grandes Expositions, universelles ou coloniales ${ }^{48}$.

Le contexte général du développement d'un intérêt pour le savoir anthropologique dans l'entre-deux-guerres est celui de la nécessité d'une légitimation nouvelle de la domination coloniale. Albert Sarraut, ancien ministre des colonies et théoricien de la colonisation, n'hésite pas, lors du congrès de la Société indigène pendant l'Exposition coloniale de Paris, en 1931, à parler « de la crise morale, de la crise de domination, de la crise d'autorité » que connaît la colonisation. Sa légitimité se voit en effet remise en cause, notamment du fait du développement de mouvements nationalistes en Asie (dans les Indes britanniques et hollandaises, mais aussi en Indochine française), au Maghreb, des revendications des nouvelles élites urbaines au Sénégal et au Dahomey, et de la propagande anticoloniale de l'Internationale communiste, comme des critiques venues des États-Unis, d'Allemagne ou d'Italie, puissances exclues du jeu colonial.

47. En 1930 encore, le gouverneur général Jules CARDE, cité dans L'Afrique française, 1930, p. 14, déclare devant le conseil général de l'AOF : "Si l'on admet qu'il existe des peuples évolués et des races attardées et qu'il est d'intérêt général que celles-ci s'élèvent au niveau de celles-là, le colonialisme se trouve implicitement justifié dans son principe. »

48. Greenhalgh, 1988. Sur l'Exposition coloniale de 1931, où dominent d'autres mises en scène, voir L'Estolle, 2000. 
Sarraut, alors ministre des Colonies, affirme en 1932, dans son discours de rentrée, devant les élèves de l'École coloniale :

«Les temps où la force seule a pu régner sont révolus [...] Nous ne devons pas opprimer, nous devons convaincre. Convaincre de quoi? De l'utilité suprême de notre tutelle, de notre supériorité scientifique et morale, de l'humanité profonde de nos desseins ${ }^{49}$. »

Un tel discours fait écho à l'analyse wébérienne de la domination : celle-ci ne dépend pas seulement du rapport de forces, elle s'enracine aussi dans la croyance à son caractère légitime. C'est très précisément la nécessité d'une légitimation fondée sur la démonstration qu'il affirme, face à «l'opinion publique» française mais surtout face à «l'opinion internationale ». La nouvelle configuration de concurrence internationale est résumée en 1931 par Sarraut, lors du congrès de la Société indigène : «Une censure internationale nouvelle fixe sur le fait colonial des regards autrement attentifs que jadis. » Cette pression internationale est particulièrement forte dans le cas des territoires sous mandat, pour lesquels les puissances tutélaires doivent rendre compte devant la Société des nations. L'ethnographie, en tant qu'elle prend pour objet privilégié les populations les plus «primitives », permet d'expliquer les difficultés des transformations que cherchent à réaliser les colonisateurs et de justifier les « retards » ou les « écarts » par rapport à l'idéal proclamé.

Mais la légitimation par la science s'adresse aussi aux colonisés euxmêmes. Selon le géographe Édouard de Martonne, auteur d'un ouvrage présentant Le Savant colonial, la science dresse aussi une barrière face aux revendications des nouvelles élites indigènes scolarisées :

« À l'heure où, dans presque toutes les colonies, s'éveillent les revendications des élites indigènes grisées de leur jeune savoir comme d'un vin nouveau, il est sage de tempérer cette présomption trop hâtive par la démonstration de notre supériorité scientifique, seule susceptible peut-être d'enchaîner les esprits sans les comprimer: il faut persuader ces écoliers de la civilisation que le degré d'émancipation croît naturellement avec le degré de culture, et que rien ne peut remplacer la maturité apportée par la science $[\ldots]^{50}$.»

Dans ce contexte, la seule pratique d'une activité scientifique, quel que soit son objet, prend un sens de justification d'une position dominante ${ }^{51}$.

49. Cité dans la revue Outre-mer, vol. III, 1932 (souligné par moi). Voir aussi HardY, 1932.

50. Martonne, 1930.

51. Dans un livre de 1916 destiné aux écoles d'AOF, cité in ConkLin, 1997, p. 136, on pouvait lire que les Français «plus avancés en civilisation » aidaient les Africains en partageant avec eux les fruits de la science, «œuvre des Blancs », mais que les Noirs pouvaient étudier pour en tirer profit. 
Ce qui est important, dans une telle perspective, c'est donc moins l'utilité directe de la pratique scientifique que le fait même qu'elle apparaisse comme une démonstration de la supériorité des colonisateurs, donc du fait que leur domination est légitime, parce qu'elle a la raison de son côté ${ }^{52}$.

C'est dans ce même contexte d'interrogation sur la légitimité de la domination coloniale qu'un certain nombre de réformateurs prônent une attention plus grande aux sociétés indigènes, et une politique plus volontariste de la métropole. Dès 1921, Maurice Delafosse (1870-1926), devenu, après une longue carrière coloniale en Afrique occidentale, professeur d'ethnographie et de langues africaines à l'École coloniale, propose ainsi un programme pour une « orientation nouvelle de la politique indigène dans l'Afrique noire ${ }^{53} »$, qui s'ouvre par un «examen de conscience » : « Nous ressentons en effet la nécessité d'excuser, ou tout au moins de justifier des actes qui, à parler net, ont consisté de notre part à déposséder des peuples de leur indépendance au profit de notre pays. »

Récusant la priorité donnée aux objectifs économiques, Delafosse dresse un bilan critique des réalisations coloniales et établit comme critère principal de légitimité de l'action coloniale française ses effets pour les populations dont elle a la «tutelle»: «Il est absolument nécessaire que notre intervention soit pour elles une cause et un élément de progrès et de bonheur, sans quoi ce serait la condamnation de toute tentative colonisatrice. » Cette question lui sert de pierre de touche pour évaluer la politique coloniale :

« Nous avons à nous demander si la politique que nous avons pratiquée jusqu'à ce jour vis-à-vis des indigènes est bien celle qui doit favoriser l'évolution rationnelle des sociétés noires vers le mieux-être ou si au contraire, elle ne risque pas de désagréger ces sociétés et de les mener à la ruine. »

On trouve ici une autre dimension fondamentale de la légitimation de l'État moderne : l'affirmation qu'il exerce sa domination en vue du bienêtre et du progrès des populations qu'il domine ${ }^{54}$. Ce qui justifie l'État colonial, c'est d'abord qu'il apporte le Progrès aux territoires et aux populations dominés (on commence alors à parler de «développement »). À côté du thème prépondérant de la «mise en valeur » économique des colonies, qui justifie la présence européenne par la nécessité économique de tirer parti des «richesses naturelles », minières et agricoles, inexploitées par les habitants des pays colonisés, c'est aussi, de plus en plus, le souci des indigènes qui apparaît comme légitimant l'action colonisatrice ${ }^{55}$.

52. Cette revendication fait parfois l'objet de violentes contestations, voir L'EstolLE, $1997 b$.

53. Delafosse, 1921.

54. Foucault, 1996.

55. Conklin, 1997. 
C'est précisément à la croisée de ces deux types de préoccupation (pour la rationalisation de la domination et le bonheur des populations) que s'inscrit l'investissement dans les sciences de l'homme, dont le projet est celui d'une connaissance rationnelle des comportements humains. Dans le cadre colonial, l'étude scientifique des indigènes apparaît comme un moyen privilégié, aux côtés de l'œuvre dans le domaine de la santé et de l'éducation, pour démontrer à la fois « l'humanité profonde » (la préoccupation pour les populations indigènes et leurs mœurs) et la « supériorité scientifique » de la puissance tutélaire.

Ce qui est nouveau, ce n'est donc pas l'idée, déjà présente au XIX siècle, que l'expansion coloniale doit s'appuyer sur l'activité scientifique. Mais c'étaient alors essentiellement les sciences participant à l'inventaire des richesses de la nature tropicale, sciences naturelles, botanique, géographie, géologie, qui étaient mobilisées ${ }^{56}$. Au début du $\mathrm{xx}^{\mathrm{e}}$ siècle, la médecine tropicale, qui met l'accent sur les soins médicaux des populations, se développe considérablement, et avec elle l'anthropologie physique que pratiquent nombre de médecins coloniaux. Le nouvel intérêt pour l'ethnologie correspond cependant à un accent nouveau mis sur la connaissance des populations et l'étude de leurs traditions. Pour Delafosse, la «politique indigène » doit être guidée par le respect des institutions et des traditions indigènes. On oublie trop souvent que Delafosse, qui plaide pour la reconnaissance des «civilisations africaines ${ }^{57}$ », est un des fondateurs, aux côtés de Marcel Mauss, de Lucien Lévy-Bruhl et de Paul Rivet, de l'Institut d'ethnologie.

Ainsi, l'appui de l'État au développement des institutions destinées à la connaissance scientifique des populations prend également sens dans les nouvelles exigences de légitimation de la domination coloniale de la France.

\section{LES ENJEUX DE LA RATIONALISATION}

C'est cette double dimension de la «domination rationnelle»recherche d'instruments plus efficaces et légitimation par la « démonstration de la supériorité scientifique » — qu'on peut lire dans les nombreux projets de rationalisation coloniale qui fleurissent alors. Un certain nombre de réformateurs défendent l'idée que, face aux nouveaux défis, le temps de «l'improvisation» est dépassé, et que la politique coloniale doit se faire

56. BonNeuil, 1999.

57. Delafosse, 1925. 
« scientifique ». Brévié plaide ainsi pour une rationalisation des méthodes de la colonisation, qui passe par une alliance avec la science :

«La mise en valeur des colonies pose des problèmes généraux et techniques d'une ampleur telle que pour les embrasser et les résoudre, il ne faut plus seulement s'abandonner aux solutions inspirées des circonstances, se livrer au génie de l'improvisation, aux initiatives trop libres de l'empirisme. La colonisation devient affaire de méthode, de calcul, de prévision, et pour tout dire, de science. Elle reste [...] un art politique et psychologique, mais qui doit être guidé, éclairé, par des données scientifiques exactes ${ }^{58}$. »

Dans cette combinaison idéale des qualités politiques et psychologiques du chef avec la rationalité scientifique, Brévié réserve une place privilégiée à la «science de l'homme », c'est-à-dire à la connaissance systématique des caractéristiques des indigènes.

Ce thème apparaît aussi dans l'avant-propos du premier numéro de la revue Outre-mer, par le gouverneur général en retraite Ernest Roume, qui affirme l'urgence de l'étude des populations indigènes devant les remises en cause de la domination ${ }^{59}$ :

«Des courants nouveaux d'idées, de sentiments, de passions se sont formés et atteignent les masses jusqu'alors inertes des populations coloniales même les plus attardées et les relations qui naissent des contacts entre des civilisations si différentes s'en trouvent singulièrement compliquées. De là résulte, plus impérieusement que jamais, la nécessité pour les représentants de la race parvenue à un degré supérieur de civilisation de porter tout leur effort sur l'étude attentive des caractéristiques des races moins évoluées, de se rendre compte le plus exactement possible des réactions, souvent si imprévues, que produisent sur elles les mesures prises à leur égard, de comprendre en un mot, et dans le sens le plus général du terme, l'âme indigène. »

La volonté de comprendre «l'âme indigène » est en même temps une réaffirmation de toute la distance, en termes d'évolution, entre le sujet et l'objet de l'ethnographie.

Ce qui rapproche ces hommes, mais aussi Delafosse ou Labouret, son successeur, qui reprend son plaidoyer pour une nouvelle politique indigène ${ }^{60}$, c'est leur lien avec l'École coloniale ${ }^{61}$. Celle-ci joue en effet un rôle central, à la fois dans le développement d'un intérêt pour l'étude des indigènes, et dans l'élaboration de projets de réforme coloniale. Cette

58. « Science et colonisation », repr. in BRÉvié, 1936.

59. Roume, 1929. Voir Piriou, 1997.

60. LABOURET, 1931.

61. Jules Brévié, comme Félix Éboué, en est ancien élève, Ernest Roume président de son conseil d'administration. Sur l'École coloniale, voir Cohen, 1973, et Dimier, 1999. 
double orientation s'exprime dans la création par son directeur, Georges Hardy (1884-1972), d'une « revue générale de colonisation », Outre-mer, dont le programme est d'insuffler à la colonisation un «esprit scientifique de bon aloi ».

On peut ici reprendre l'analyse de Weber sur l'intérêt à la rationalisation des agents de la bureaucratie. Il note que « de plus en plus le savoir spécialisé de l'expert est devenu le fondement de la position de pouvoir de l'occupant d'un poste ${ }^{62} \gg$. La possession d'un savoir spécifique est une ressource dont disposent les hommes de l'administration, qui leur permet de défendre leur autonomie contre le monarque ou le ministre : la connaissance des dossiers fonde leur compétence et légitime leur position. La qualification de l'agent bureaucratique, certifiée par une formation spécifique, est donc à la fois un instrument de contrôle de l'accès aux postes (contre la faveur du prince), et une garantie contre l'arbitraire du pouvoir politique.

Une telle analyse éclaire le rôle central de l'École coloniale dans la promotion de la connaissance scientifique des indigènes. Celle-ci accompagne, en effet, un travail de rédéfinition du statut des principaux agents de l'État colonial : les administrateurs des colonies. Décidés à lutter contre la réputation peu flatteuse des coloniaux (qui se plaignent d'être vus comme des «aventuriers »), un certain nombre d'hommes liés à l'École coloniale ont adopté une stratégie de «professionnalisation» et de légitimation par la compétence, et valorisent le «métier » et la «vocation » d'administrateur. C'est en mettant en avant la nécessité d'une formation spécialisée des administrateurs que l'École coloniale est progressivement parvenue à acquérir le monopole de l'accès au corps des administrateurs des colonies ${ }^{63}$.

Ce groupe tend à se constituer sur le modèle de ce que Weber appelle un « groupe statutaire », caractérisé par un fort esprit de corps et une intériorisation de valeurs « héroïques ». Ce thème est développé dans les différents textes qui cherchent à définir — et, par là même, à faire exister — cet ethos des administrateurs coloniaux, et une sorte de «charisme de fonction », depuis les interventions au $\mathrm{I}^{\mathrm{er}}$ congrès professionnel des Administrateurs coloniaux, en 1931, jusqu' au livre de Robert Delavignette, Les Vrais Chefs de l'Empire, hymne à la gloire des administrateurs. Dans une section intitulée «Organisation héroïque du monde», Delavignette (1897-1976),

62. WEBER, 1968, vol. II, p. 994.

63. On a parfois tendance à utiliser comme interchangeables les termes «administrateur» et « agent de l'administration ». En fait, le titre d'administrateur des colonies est réservé aux seuls membres du corps, devant être depuis 1912 brevetés de l'École coloniale, qui formait auparavant seulement un quart des administrateurs. Les « adjoints des services civils », recrutés sur place, doivent désormais y faire un stage d'un an avant d'entrer dans le « corps ». 
directeur de l'École coloniale de 1937 à 1947, tente de définir ce qui fonde la «supériorité héroïque » de l'administrateur). C'est à la fois sa personnalité de «chef», son « caractère », obtenu par un travail sur soi constant, mais aussi la possession d'un savoir spécifique, qui le distingue à la fois des colonisés et des autres coloniaux : «Le colonial est un homme qui sait plus de choses que les indigènes du pays colonisé, et qui en sait mieux le prix que ces autres indigènes, ceux du pays colonisateur ${ }^{64}$. »

Les administrateurs revendiquent un domaine de compétence spécifique : ils se veulent avant tout les «spécialistes » des indigènes. Lors du congrès de l'Association amicale des administrateurs coloniaux, tenu en 1931 lors de l'Exposition coloniale, Hubert Deschamps (1900-1979), alors jeune administrateur ${ }^{65}$, évoque l'importance nouvelle des «services techniques» que doit coordonner l'administrateur et affirme que «le développement des diverses techniques nous en laisse cependant une qui nous appartient en propre et qui conditionne toutes les autres. L'Administrateur est le spécialiste de la politique indigène ». Plus loin, il ajoute : «Pour bien faire son métier, il faut l'aimer. Et notre métier à nous, c'est l'indigène ${ }^{66}$. »

Alors que la compétence des administrateurs des colonies était jusque-là fondée sur la maîtrise d'un savoir proprement administratif, conçu comme universel (le droit), la définition de l'administrateur comme « spécialiste des indigènes » amène à transformer la formation que reçoivent les futurs administrateurs, auparavant essentiellement juridique ${ }^{67}$. Hardy met l'accent sur les savoirs spécifiques qui portent sur les « milieux indigènes »: il renforce les cours de géographie humaine, ethnologie, langues indigènes, droit coutumier, et crée un cours de «psychologie appliquée à la colonisation ». C'est aussi sous sa direction que s'effectue un rapprochement entre l'Institut d'ethnologie et l'École coloniale.

Le plaidoyer pour une rationalisation de l'action coloniale est aussi un plaidoyer pro domo, pour ceux qui s'affirment comme les agents privilégiés de la rationalisation. Une définition en tant que «spécialiste des indigènes », voire de leur «défenseur », est aussi une revendication pour un contrôle plus grand du corps des administrateurs des colonies sur la détermination des objectifs et des moyens de la politique coloniale, vis-à-vis de tous ceux qui menacent leur autonomie : le pouvoir politique métropolitain (en particulier les parlementaires), le ministère des Colonies (dont le titu-

64. Delavignette, 1946, p. 53 (souligné par moi).

65. Premier de sa promotion à l'École coloniale en 1924, Hubert Deschamps est un des exemples de double carrière, d'abord coloniale, puis universitaire : il sera, jusqu'en 1970, directeur du centre d'études africaines de la Sorbonne.

66. Deschamps, 1931.

67. CoHEn, 1973. 
laire est toujours un «politique » et non un « colonial ${ }^{68}$ ), mais aussi les spécialistes du droit colonial, pour qui les administrateurs ne sont que des exécutants censés appliquer des règles valides universellement, les représentants des grands intérêts économiques coloniaux et les colons, sans oublier les élites colonisées.

Comme l'affirme en 1931 Hardy, en conclusion du congrès de la Société indigène : «Il faut [...] imposer à la colonisation une allure scientifique, une action fondée en raison qu'elle n'avait pas eue jusqu'ici et qu'elle est portée à dédaigner ${ }^{69}$. » Cette formulation indique bien le caractère militant, voire polémique, de la revendication de rationalité : la rationalisation n'apparaît pas ici comme un processus impersonnel, mais s'inscrit au contraire dans les luttes de concurrence internes à l'entreprise de colonisation. Les administrateurs coloniaux, alors en position de relative faiblesse, mobilisent la science comme alliée dans cette lutte pour la rationalisation.

DIVISION DU TRAVAIL ET AUTONOMISATION

Cette double transformation, à la fois des conditions de la domination coloniale et de la profession d'administrateur, constitue l'arrière-plan sur lequel se noue dans l'entre-deux-guerres, autour d'un intérêt commun à la rationalisation, une alliance entre certains représentants du monde savant et les partisans d'une réforme « rationnelle» des pratiques coloniales. Brévié théorise cette alliance nécessaire entre des spécialistes de la science et une administration coloniale rationnelle. Pour lui, la rationalisation implique aussi une spécialisation et une division du travail entre chercheurs et hommes d'action.

«Ainsi de plus en plus la colonisation a besoin de savants, de chercheurs impartiaux et désintéressés qui voient à distance, hors de l'urgence et du feu de l'action. Il lui faut des laboratoires mieux outillés, des Instituts de recherche; il lui faut appuyer son action sur une véritable culture scientifique coloniale, s'inspirer de la méthode expérimentale, recevoir les directives d'une science précise et pratique ${ }^{70}$.»

Il est remarquable que Brévié évoque précisément les qualités d'impartialité et de désintéressement que revendiquent les chercheurs. Un tel pro-

68. C'est-à-dire non membre du corps. La seule exception est celle de Brévié, ministre d'avril 1942 à mars 1943.

69. HARDY, 1932.

70. BRÉVIÉ, 1936. 
gramme rencontre l'adhésion du monde savant, en particulier de Paul Rivet, engagé dans un projet de rationalisation et de systématisation de la science de l'homme ${ }^{71}$. Si l'ethnographie existe en tant que pratique savante coloniale, l'ethnologie, en tant que discipline universitaire n'est alors que virtuelle, et dépend de l'appui de l'État colonial pour se développer. Une telle alliance va permettre le succès d'un certain nombre d'initiatives institutionnelles, généralement évoquées comme autant de noms de victoires dans la chronique de l'institutionnalisation de l'ethnologie en France: outre la création de l'Institut d'ethnologie, on peut mentionner celle de la Société des africanistes (1930), l'appui financier à la mission DakarDjibouti, dirigée par Marcel Griaule (1931-1932) — tous deux dans le cadre de la préparation de l'Exposition coloniale —, et enfin la création du musée de l'Homme (1937) qui, comme le rappellera Rivet en demandant la présence de troupes coloniales lors de son inauguration, se voulait d'abord un «grand musée colonial ».

La nécessité de manifester l'intérêt scientifique de l'État pour les populations coloniales est constamment évoquée pour justifier le financement public d'initiatives institutionnelles dans le domaine de l'ethnologie. Ainsi, en 1930, le ministre des Colonies écrit au gouverneur général de l'AEF pour lui demander d'appuyer la mission Dakar-Djibouti :

« À l'heure où l'Exposition coloniale de 1931 attire l'attention du grand public et des savants étrangers sur l'activité coloniale de la France, il est indispensable de montrer aux éléments avertis de ces questions que les pouvoirs publics ne négligent rien pour en connaître et emploient dans ce but des méthodes modernes qui n'ont rien à envier à celles des instituts des autres nations. »

Une telle phrase donne une des clés de l'appui considérable que reçoit le programme de l'ethnologie scientifique telle que l'incarne Rivet: le contexte de concurrence internationale dans la démonstration d'un intérêt scientifique pour les populations colonisées pousse à favoriser l'investissement dans les institutions qui, tels l'Institut d'ethnologie ou le musée d'Ethnographie du Trocadéro, apparaissent, en vertu de leur capacité de mobilisation d'un important capital scientifique, comme les mieux placés pour défendre les couleurs de la «science française ». C'est donc ici le besoin d'une légitimation sur la scène internationale qui constitue le moteur essentiel de la délégation du travail de connaissance des indigènes à des spécialistes «professionnels », ayant une autonomie institutionnelle à

71. Professeur au Muséum national d'histoire naturelle, secrétaire général de l'Institut d'ethnologie, directeur du musée d'Ethnographie, Paul Rivet cumule les fonctions scientifiques. Son projet est d'unifier les différentes branches de la «science de l'homme » sous la bannière de «l'ethnologie », voir L'Estolle, 1997a. 
l'égard de l'administration coloniale, plutôt qu'à des administrateurs provisoirement détachés pour des travaux de recherche.

L'aiguillon de la concurrence internationale apparaît nettement lors du congrès des Sciences coloniales de 1937, au cours duquel Maurice Leenhardt déclare, à propos de la nécessité d'organiser la recherche dans les possessions françaises d'Océanie :

«Toute la bibliographie de ces domaines français est en anglais ou en allemand. L'exemple le plus remarquable est aux Nouvelles-Hébrides : aux travaux anglais de Rivers, de Deacan, etc., ou allemands, de Speiser, l'édition je ne dis pas la science — l'édition française n'a pas à opposer un seul volume, hors le roman de P. Benoît, «Eromango ». Ce n'est pas suffisant pour persuader les Anglais, qui luttent d'influence dans cet archipel, que nous connaissons ces îles mieux qu'eux ${ }^{72}$.»

La connaissance scientifique apparaît ainsi comme un titre de possession. Réaliser un travail de connaissance scientifique d'un territoire, c'est aussi acquérir un droit moral à sa domination, que l'on peut opposer aux autres nations.

L'appui des gouvernements coloniaux aux institutions scientifiques métropolitaines se justifie dans une double perspective : il s'agit à la fois de former les futurs cadres coloniaux, et de jouer un rôle d'organisation et d'animation de la recherche dans les colonies. De fait, les savants des institutions centrales se donnent, dans un premier temps, essentiellement un rôle de centralisation et de rationalisation de la production des données; à côté des expéditions scientifiques, le rôle des agents coloniaux dans la collecte des informations sur le terrain reste essentiel ${ }^{73}$.

Cependant, surtout après 1945, se dessine progressivement une nouvelle division du travail, allant dans le sens d'une «spécialisation fonctionnelle », avec une distinction croissante parmi les agents de l'État des fonctions de chercheur de celles d'administrateur ${ }^{74}$. Ce mouvement, qui aboutit à la constitution d'un corps de professionnels du savoir ethnologique payés par l'État, provoque un certain nombre de transformations, dont les termes de «professionnalisation » et « institutionnalisation » masquent trop souvent la complexité. Ce nouveau modèle de division du travail entre étude

72. LeENhardt, 1938, p. 528.

73. Ainsi, le musée d'Ethnographie et l'Institut d'ethnologie utilisent les réseaux de l'administration coloniale pour la collecte de «données » diverses, qu'elles soient d'ordre linguistique, ethnographique, ou d'objets indigènes, voir L'EstoILE, 1999.

74. Le phénomène de spécialisation fonctionnelle, avec la définition de «sphères de compétence spécifiques », est une autre dimension du processus de division rationnelle du travail qui caractérise la domination bureaucratique. On peut, en prolongeant l'analyse de Weber, considérer que l'autonomisation d'un appareil de production des connaissances ne fait que pousser à bout cette logique de division fonctionnelle. 
et administration, à la fois de spécialisation et de complémentarité, est affirmé dans un des premiers manuels universitaires d'ethnologie, significativement appelé Ethnologie de l'Union française. L'ouvrage, publié en 1953 aux Presses universitaires de France, est cosigné par André LeroiGourhan, alors maître de conférences en ethnologie coloniale à Lyon, et Jean Poirier, professeur à l'École nationale de la France d'outre-mer (nouveau nom de l'École coloniale) ${ }^{75}$.

On retrouve dans cet ouvrage universitaire la double thématique de l'instrument de politique indigène et de la légitimation. La quatrième partie de l'ouvrage, "Union française et ethnologie », est ainsi introduite par un court texte, au titre significatif, «Intérêt national et devoir international » :

«C'est l'ethnologie seule qui peut fonder valablement une politique. Il serait vain de prétendre conseiller et diriger une société indigène sans procéder à l'étude de ses mœurs et de sa mentalité; la connaissance des races, de la culture matérielle et des genres de vie, des langues, des structures familiale et politique, des croyances religieuses, l'étude systématique de la psychologie sont les conditions mêmes de l'action ${ }^{76}$.»

La continuité avec l'argumentation des administrateurs promouvant l'ethnographie comme modalité de déchiffrement des populations indigènes est frappante ${ }^{77}$. Le deuxième argument est tout aussi remarquable :

«Mais si la connaissance des données ethnologiques s'avère indispensable pour mener à bien toute action outre-mer, l'on doit remarquer que cette connaissance répond désormais à des obligations d'ordre international. En effet, la France s'est engagée à prendre toutes mesures nécessaires au développement des cultures indigènes; elle s'est engagée à respecter l'originalité des sociétés d'outre-mer; tout cela implique un effort orienté vers une connaissance toujours meilleure des communautés prises en charge. En ce qui concerne les anciens mandats de la Société des nations, les engagements souscrits sont encore plus précis et les gouvernements responsables doivent périodiquement rendre compte de leur action. D'une manière générale, l'on peut dire qu'à partir du moment où les autorités françaises ont pris en charge une population d'outre-mer, leur responsabilité se trouve engagée non seulement pour la mise en valeur matérielle du pays, mais encore pour la mise en valeur des hommes : l'étude des coutumes, l'exploration archéologique et historique, l'analyse de l'art, de la littérature, de la pensée philosophique, toute cette recherche ethnologique fait partie du devoir des autorités de tutelle ${ }^{78}$.

75. Ces deux personnages sont de futurs «grands noms» de la discipline : le premier régnera sur la préhistoire et les études de «technologie» en France; le second dirigera le volume consacré à l'ethnologie dans l'« Encyclopédie de la Pléiade », voir PoIRIER, dir., 1968.

76. Leroi-Gourhan et PoIRIER, 1953, vol. II, p. 897-898.

77. On note la parenté avec les formulations indigénistes étudiées par Claudio LomNITZ et par Antonio Carlos DE SouzA Lima, infra, respectivement p. 345-380 et 381-410, dans ce volume.

78. Leroi-Gourhan et Poirier, 1953, vol. II, p. 897-898 (souligné par moi). 
Cette double justification de l'importance de la recherche ethnologique, par l'intérêt national et le devoir international, est ici encore fortement reliée à la fois aux objectifs de réforme coloniale et à la transformation des rapports de domination coloniaux, avec l'émergence de l'Union française, qui prétend instaurer une nouvelle forme de relations, plus égalitaires, entre la métropole et les territoires d'outre-mer ${ }^{79}$, et une pression internationale plus forte encore que dans l'entre-deux-guerres. Le contexte évoqué ici est celui de l'obligation faite à l'État colonial français de « rendre compte de [son] action » sur le plan international, en particulier pour les anciens pays de mandat, Cameroun et Togo, devenus des «territoires associés » sous le contrôle de l'ONU. C'est encore la nécessité de légitimation de l'action coloniale qui est ici mise au premier plan : l'ethnologie apparaît comme un «gage» de «bonne volonté » authentifiant le respect de l'État français pour les cultures indigènes. La notion de «mise en valeur des hommes », présentée comme contrepartie de la «mise en valeur matérielle », fait écho à l'argument de Lévy-Bruhl justifiant la création de l'Institut d'ethnologie et, au-delà, à la valorisation par les administrateurs réformistes de la «dimension humaine » de la colonisation.

La relation entre administration coloniale et ethnologie, suggérée par Leroi-Gourhan et Poirier, est celle d'une complémentarité et d'un appui mutuel : «l'action coloniale » doit s'appuyer sur les ethnologues; ceux-ci jouent ainsi le rôle d'un «service extérieur » de l'État, qui effectue en quelque sorte une délégation du travail de connaissance aux chercheurs professionnels ${ }^{80}$. Cet idéal d'une connaissance au service de l'action coloniale, élaborée dans la discussion entre administrateurs et chercheurs, entre cependant en contradiction croissante avec d'autres exigences. En se définissant comme « spécialistes des sociétés et des mentalités indigènes », les ethnologues entrent en concurrence directe avec les administrateurs coloniaux sur le terrain même que ceux-ci cherchaient à occuper. On pourrait caractériser leurs relations comme correspondant à un modèle d'interdépendance concurrentielle, marqué par les conflits d'intérêts et de frontières.

79. La constitution de 1946 donnait le nom d'Union française à l'ensemble formé par la France métropolitaine et les pays d'outre-mer, étendant la citoyenneté française (quoique avec des différences de statut) à tous les ressortissants de l'Union.

80. Le programme d'une « sociologie des peuples dépendants », développé à l'Institut français d'Afrique noire à la fin des années quarante par de jeunes chercheurs comme Georges Balandier et Paul Mercier, qui prévoit une définition des objectifs de recherche en collaboration entre administrateurs et chercheurs, est l'élaboration théorique de cette situation d'interdépendance structurelle entre deux groupes de fonctionnaires désormais conçus comme distincts, voir Mercier, 1951; Balandier et Pauvert, 1952. 
De plus en plus, cependant, le renforcement des institutions scientifiques métropolitaines ${ }^{81}$, ainsi que l'effort des ethnologues pour être reconnus dans l'establishment savant — et, au-delà, dans l'univers intellectuel métropolitain, créent les conditions d'une divergence croissante des objets et des formes du savoir : les caractéristiques exigées pour qu'une production soit reconnue comme ethnologique se différencient de plus en plus des exigences du savoir administratif, lui-même de plus en plus tourné vers de nouvelles questions, notamment économiques ${ }^{82}$. La relation de concurrence essentielle se déplace : elle apparaît désormais au sein de l'espace scientifique qui se constitue alors, entre des individus établis dans des institutions de recherche coloniale (IFAN ou ORSTOM) et d'autres qui, dépendant d'institutions de recherche métropolitaines, doivent justifier leur statut dans l'espace scientifique métropolitain. La revendication de la «pureté scientifique » est mobilisée dans les luttes scientifiques; elle aboutit notamment à la stigmatisation des recherches disqualifiées comme «appliquées », par contraste avec la «science pure » et désintéressée ${ }^{83}$.

La notion de «domination rationnelle » permet de dépasser le postulat d'une antinomie radicale et immuable entre raison savante et raison d'État. Elle fournit un outil pour analyser la parenté entre des formes de rationalité administrative et de rationalité scientifique, à condition d'entendre cette notion non seulement au sens de rationalisation formelle des techniques de domination, mais aussi au sens de recours à un mode de légitimation spécifique, caractérisé par la mise en avant du caractère fondé en raison de la domination. Le thème wébérien de la «domination par le savoir » prend ainsi une double signification: la bureaucratie bénéficie d'un côté de la rationalisation des instruments de connaissance, et de l'autre de la caution de rationalité que lui apporte la science. C'est entre ces deux pôles que s'inscrivent les relations complexes, et parfois paradoxales, entre la connaissance savante des populations et leur administration. Ces deux dimensions de la domination rationnelle ne sont d'ailleurs pas indépendantes : l'utilisation des instruments « rationnels » de la science contribue à

81. Création en 1944 d'une section «Ethnologie et préhistoire » au Centre national de la recherche scientifique, en 1947 de la VI ${ }^{\mathrm{e}}$ section de l'École pratique des hautes études, et en 1958 d'une chaire d'anthropologie sociale et culturelle au Collège de France, voir GAILLARD, 1989.

82. COOPER, 1997

83. Cette situation crée les conditions d'un conflit de valeurs, pour les ethnologues coloniaux les plus ambitieux scientifiquement, qui apparaît nettement dans les premiers travaux de Balandier et Mercier. Les chercheurs « professionnels » défendent à l'égard de l'administration leur «autonomie », c'est-à-dire le monopole de la définition des méthodes et des objets pertinents. 
produire la croyance dans la possibilité de maîtriser une réalité complexe, qui légitime la domination aux yeux mêmes de ceux qui l'exercent.

La notion de «domination rationnelle » permet aussi de faire apparaître que la relation entre l'ethnologie en tant que discipline universitaire et l'administration coloniale en tant qu'institution, généralement prise comme point de départ de la réflexion sur ces questions et projetée rétrospectivement, doit au contraire être considérée comme un «cas particulier », c'està-dire une configuration historique singulière dans les rapports entre production de savoir sur les populations indigènes et des pratiques de gestion des populations indigènes ${ }^{84}$. La particularité de cette configuration relativement récente est que ces deux ensembles de pratiques sont réalisés dans des institutions, et par des agents, distincts.

Si l'État colonial contribue au développement d'une approche scientifique des populations indigènes, ce n'est pas en vertu d'un processus de rationalisation inexorable; ce projet apparaît plutôt comme «porté » (au sens où Weber parle de groupes « porteurs » d'un message religieux) par la conjonction de divers groupes liés à l'État partageant à la fois un « intérêt à la rationalisation » et un intérêt pour la connaissance des indigènes. Le souci d'une connaissance savante des populations indigènes et de leurs coutumes, qu'incarne l'ethnologie, apparaît ainsi à la fois comme un élément clé et un symbole d'une nouvelle technologie politique, fondée sur la prise en compte de la différence des civilisations. Dans une configuration marquée à la fois par une remise en cause de la domination coloniale et par une forte pression internationale, l'ethnologie apparaît comme permettant de conjuguer trois facteurs de légitimation : l'exercice d'une activité scientifique, la démonstration d'un intérêt pour les indigènes et le respect de leurs traditions.

Ces intérêts partagés fournissent, dans l'entre-deux-guerres, le fondement d'une alliance entre les promoteurs de projets de rationalisation de la domination coloniale - qui accompagnent une redéfinition du rôle d'administrateur comme «spécialiste des indigènes » —, et des savants qui dépendent de l'appui de l'État colonial pour l'existence de leurs institutions et de ses agents pour la collecte des données. On peut ainsi analyser la relation entre administration coloniale et ethnologie, telle qu'elle émerge au cours du processus d'institutionnalisation, comme une forme d'interdépendance concurrentielle entre plusieurs groupes liés à l'État, interdépendance dont les conditions se modifient au cours du temps.

Cette alliance, sur la base d'une division du travail fondée sur une spécialisation fonctionnelle (les spécialistes du savoir fournissant des informa-

84. L'ensemble des contributions à ce volume permet de saisir la variété de ces configurations. 
tions, mais aussi des cadres d'interprétation, aux spécialistes de l'action sur les indigènes), rend possibles un certain nombre de créations institutionnelles, qui elles-mêmes créent les conditions du développement d'un nouveau corps de spécialistes des populations indigènes, les ethnologues, et aident à sa reconnaissance dans le monde académique et intellectuel. À son tour, le développement d'une base institutionnelle relativement indépendante de l'État colonial - ce qui explique que la discipline ait pu lui survivre - favorise une redéfinition de la discipline, à travers des conflits internes qui mettent en danger la complémentarité d'abord espérée entre préoccupations coloniales et savantes. À cela s'ajoute la remise en question croissante de la domination coloniale dans les milieux intellectuels métropolitains, qui rend désormais difficile de proclamer la place centrale de l'ethnologie dans la «mise en valeur » coloniale, affirmée par Lévy-Bruhl dans l'entre-deux-guerres. Le rapport fondateur avec la gestion des populations indigènes devient invisible dans ce qui s'affirme comme la nouvelle science de l'homme, revendiquant l'héritage des Lumières.

Benoît DE L'EsToILE

(mars 2000). 


\section{LISTE DES RÉFÉRENCES}

Amselle (Jean-Loup) et M'BокоLо (Elikia), dir., 1999, Au cæur de l'ethnie. Ethnie, tribalisme et Etat en Afrique, $1^{\mathrm{re}}$ éd. 1985, ici Paris, La Découverte (La Découverte Poches, 68. Sciences sociales et humaines).

Balandier (Georges) et PAuvert (Jean-Claude), 1952, Les Villages gabonais. Aspects démographiques, économiques, sociologiques. Projets de modernisation, Montpellier, Impr. de la Charité (Mémoires de l'Institut d'études centrafricaines).

Bazin (Jean), 1998, « À chacun son Bambara », in Amselle et M'Boкolo, 1999, p. $87-127$.

BonNEull (Christophe), 1991, Des savants pour l'Empire, Paris, ORSTOM.

BonNeuil (C.), 1999, «Le Muséum national d'histoire naturelle et l'expansion coloniale de la III $^{\mathrm{e}}$ République (1870-1914)», Revue d'histoire de la France d'outre-mer, t. LXXXVI, 322-323, p. 143-169.

BRÉvié (Jules), 1936, Trois études de Monsieur le gouverneur général Brévié, Dakar, Imprimerie du gouvernement général de l'AOF.

BriAn (Éric), 1994, La Mesure de l'État. Administrateurs et géomètres au XVIII siècle, Paris, Albin Michel (L'Évolution de l'humanité).

Chauleur, 1943, «Esquisse ethnologique pour servir à l'étude des principales tribus des territoires du Cameroun sous mandat français », Bulletin de la Société d'études camerounaises, 3.

Chombart de Lauwe (Paul-Henry) et Deboudaud (J.), 1939, «Carte schématique des populations du Cameroun », Journal de la Société des africanistes, p. 197203.

CoHen (William B.), 1973, Empereurs sans sceptre. Histoire des administrateurs de la France d'outre-mer et de l'École coloniale, trad. de Rulers of Empire. The French colonial service in Africa, Stanford, 1971, ici Paris, Seuil.

Conn (Bernard), 1987, « The census, social structure and objectification in South Asia », in An anthropologist among the historians, Oxford, Oxford University Press, p. 224-254.

Conklin (Alice L.), 1997, A mission to civilize. The republican idea of empire in France and West-Africa, 1895-1930, Stanford, CA, Stanford University Press.

COOPER (Frederick), 1997, "Modernizing bureaucrats, backward Africans and the Development concept », in Frederick COOPER et Randall PACKARD, dir., International development and the social sciences, Berkeley, University of California Press, p. 64-92.

Delafosse (Maurice), 1921, «Sur l'orientation nouvelle de la politique indigène dans l'Afrique noire », Renseignements coloniaux et documents publiés par le Comité de l'Afrique française, 6, p. 145-152.

Delafosse (M.), 1925, Les Civilisations négro-africaines, Paris, Larose.

Delavignette (Robert), 1946, Service africain (éd. compl. de Les Vrais Chefs de l'Empire, censuré en 1940), Paris, Gallimard. 
Deschamps (Hubert), 1931, «La vocation coloniale et le métier d'administrateur », Renseignements coloniaux, 10, p. 497-500.

Dimier (Véronique), 1999, Construction et enjeu d'un discours colonial scientifique sur l'administration coloniale comparée, vers 1930-vers 1950, thèse de doctorat, Grenoble, Université de Grenoble.

Dugast (Idelette), 1948, «Essai sur le peuplement du Cameroun », Bulletin de la Société d'études camerounaises, vol. XXI-XXII, p. 19-34.

Dugast (I.), 1949, Inventaire ethnique du Sud-Cameroun, Cahors, Impr. de A. Coueslant (Mémoires de l'Institut français d'Afrique noire. Centre du Cameroun).

Dugast (I.), 1954, «Banen, Bafia and Balom », in Peoples of the Central Cameroons, Londres, International African Institute (Ethnographic Survey of Africa).

Dugast (I.), 1955-1959, Monographie de la tribu des Ndiki (Banen du Cameroun), Paris, Institut d'ethnologie, 2 vol.

Éвоú́ (Félix), 1931, «Les peuples de l'Oubangui-Chari. Essai d'ethnographie, de linguistique et d'économie sociale », Bulletin de l'Afrique française. Renseignements coloniaux et documents, repr. Bulletin de la Société des recherches congolaises, Imprimerie du gouvernement général de l'AEF, 17, 1932.

Foucault (Michel), 1996, «La gouvernementalité », Dits et écrits, vol. III, 239, p. 635-657.

Gaillard (Gerald), 1989, «L'ethnologie avant le CNRS », Cahiers pour une histoire du CNRS, 3, p. 85-129.

Goody (Jack), 1995, The Expansive Moment. Anthropology in Britain and Africa, 1918-1970, Cambridge, Cambridge University Press.

Greenhalgh (Paul), 1988, Ephemeral vistas. The Expositions universelles, great exhibitions and world's fairs, 1851-1939, Manchester, Manchester University Press.

Hardy (Georges), 1932, Congrès international et intercolonial de la Société indigène, Exposition coloniale internationale de Paris, 5-10 oct. 1931, Paris.

« Inventaire ethnique et linguistique du Cameroun sous mandat français. Documents mis à jour au 31/12/1933 et communiqués par M. le commissaire de la République », 1934, Journal de la Société des africanistes, fasc. II.

LABOURET (Henri), 1931, À la recherche d'une nouvelle politique indigène dans l'Ouest africain, Paris, Éd. du Comité de l'Afrique française.

LEENHARDT (Maurice), 1938, «L'organisation de la recherche scientifique en Océanie française ", in Congrès de la recherche scientifique dans les territoires d'outre-mer. Exposition internationale de Paris, 1937, Paris, Association Colonie-Sciences, p. 527-533.

Leroi-Gourhan (André) et Poirier (Jean), 1953, L'Ethnologie de l'Union française, Paris, Presses universitaires de France, 2 vol.

L'Estoile (Benoît de), 1994, «L'anthropologue face au monde moderne. Malinowski et la rationalisation de l'anthropologie et de l'administration », Genèses, 17, p. 140-163.

L'Estoile (B. de), 1997a, «Africanisme et Africanism. Esquisse de comparaison franco-britannique », in Anne Piriou et Emmanuelle Sibeud, dir., L'Africanisme en questions, Paris, École des hautes études en sciences sociales, Centre d'études africaines (Dossiers africains), p. 19-42.

L'Estolle (B. de), 1997b, « Au nom des "vrais Africains". Les élites scolarisées de l'Afrique coloniale face à l'anthropologie (1930-1950)», Terrain, 28, p. 87102. 
L'Estoile (B. de), 1997c, «The "natural preserve of anthropologists". Social anthropology, scientific planning and development », Social Sciences Information, vol. XXXVI, 2, p. 343-376.

L'Estoile (B. de), 1999, «Une petite armée de travailleurs auxiliaires. La division du travail et ses enjeux dans l'ethnologie française des années $1930 »$, communication au colloque du Centre de recherches historiques sur les recherches collectives dans les sciences sociales, Paris, nov. 1999.

L'Estoile (B. de), 2000, «Des races non pas inférieures, mais différentes. De l'Exposition coloniale au musée de l'Homme », in Claude BlanCKAERT, dir., Politiques de l'anthropologie. Discours et pratiques en France (1860-1940), Paris, L'Harmattan, sous presse.

LÉvy-BruHL (Lucien), 1925, «L'Institut d'ethnologie de l'université de Paris », Revue d'ethnographie et de traditions populaires, vol. XXIII-XXIV, p. 1-4.

LÉvy-Bruhl (L.), 1929, « Rapport d'activité de l'Institut d'ethnologie pour 1929 », Annales de l'université de Paris, p. 417-422.

MaLINOwSKI (Bronislaw), 1930, « The rationalization of anthropology and administration », Africa, 3, p. 405-430.

Martonne (Édouard de), 1930, Le Savant colonial, Paris, Larose (Vies coloniales).

MAupoIL (Bernard), 1939, «L'étude des coutumes juridiques de l'AOF (étude administrative) », in Coutumiers juridiques de l'Afrique occidentale française. T. I : Sénégal, Paris, Larose (Publications du Comité d'études historiques et scientifiques de l'AOF).

MERCIER (Paul), 1951, Les Tâches de la sociologie, Dakar, Institut français d'Afrique noire.

Nordman (Daniel) et RaIson (Jean-Pierre), 1980, Sciences de l'homme et conquête coloniale. Constitution et usage des sciences humaines en Afrique $\left(X I X^{e}-X X^{e}\right.$ siècles), Paris, Presses de l'École normale supérieure.

Pels (Peter), 1996, « The pidginization of Ludguru politics. Administrative ethnography and the paradoxes of Indirect Rule », American Ethnologist, vol. XXIII, 4, p. 738-761.

PIRIOU (Anne), 1997, «Indigénisme, sciences coloniales et changement social. Le cas de la revue Outre-mer », in Anne PiRiou et Emmanuelle Sibeud, dir., L'Africanisme en questions, Paris, École des hautes études en sciences sociales, Centre d'études africaines (Dossiers africains), p. 43-69.

PoIRIER (Jean), dir., 1968, Ethnologie. Vol. I : Ethnologie générale, Paris, Gallimard (Encyclopédie de la Pléiade, 24).

Rapport annuel du gouvernement français sur l'administration sous mandat des territoires du Cameroun pour 1924, 1925, Paris, Larose.

Roume (Ernest), 1929, «Avant-propos », Outre-mer, 1, mars, p. 3-5.

Souza Lima (Antonio Carlos de), 1995, Um grande cerco de paz. Poder tutelar, indianidade e formação do Estado no Brasil, Petropolis, Vozes.

Weber (Max), 1968, Economy and society. An outline of interpretative sociology, éd. par Guenther Rотн et Claus W тттісн, Berkeley, University of California Press, 2 vol.

Weber (M.), 1971, Économie et société, publ. sous la dir. de Jacques Chavy et d'Éric De Dampierre, t. I, Paris, Plon (Recherches en sciences humaines).

Weber (M.), 1996, Sociologie des religions, éd. et trad. de l'allemand Jean-Pierre Grossein, introd. Jean-Claude PAsseron, Paris, Gallimard (Bibliothèque des sciences humaines). 